\title{
THE ASSOCIATION OF CATARACT TYPE ON CENTRAL CORNEAL THICKNESS AFTER PHACOEMULSIFICATION CATARACT SURGERY
}

\author{
Hussnain Abbas, Abdul Rauf, Shagufta Perveen, Shahid Hamid Mehmood*, Irshad Hussain**, Khalid Naimat*** \\ Combined Military Hospital Multan/National University of Medical Sciences (NUMS) Pakistan, *Combined Military Hospital Peshawar/National University of \\ Medical Sciences (NUMS) Pakistan, ${ }^{* *}$ Combined Military Hospital Murree/National University of Medical Sciences (NUMS) Pakistan, ${ }^{* * * H T E C ~ I n s t i t u t e ~ o f ~}$ \\ Medical Sciences, Taxila/National University of Medical Sciences (NUMS) Pakistan
}

\begin{abstract}
Objective: To assess any association between the type of cataract and the central corneal thickness at pre-operative and postoperative three-day and one-month appointments after phacoemulsification surgery.

Study Design: Prospective, comparative study.

Place and Duration of Study: Eye Department Combined Military Hospital Multan, from Jun 2020 to Apr 2021.

Methodology: A total of 89 patients requiring cataract surgery by phacoemulsification were recruited. Age and gender were recorded for each patient. Patient's type of cataract based on maturity was recorded before the surgery. Phacoemulsification cataract surgery using 'divide and conquer' technique was carried out. Patients' central corneal thickness was measured at the pre-operative, three-day post-operative and one-month post-operative appointment using traditional non-contact tonopachymeter.

Results: There were 49 (55.1\%) male and 40 (44.9\%) female patients with a mean age of $62.15 \pm 12.40$ years. No significant association was found between the type of cataract and central corneal thickness at the three-time intervals $(p=0.14)$. Central corneal thickness significantly increased from $0.48 \pm 0.04 \mathrm{~mm}$ pre-operatively to $0.51 \pm 0.04 \mathrm{~mm}$ at the three-day post-operative appointment $(p<0.001)$. It then reduced to $0.49 \pm 0.04 \mathrm{~mm}$ at the one-month appointment $(p=0.01)$.

Conclusion: The type of cataract is not associated with the variation in central corneal thickness due to cataract surgery. Central corneal thickness significantly increases from the pre-operative to right after the cataract surgery. However, it returns to preoperative levels after a one-month period.
\end{abstract}

Keywords: Cataract type, Central corneal thickness, Phacoemulsification surgery.

How to Cite This Article: Abbas H, Rauf A, Perveen S, Mehmood SH, Hussain I, Naimat K. The Association of Cataract Type on Central Corneal Thickness After Phacoemulsification Cataract Surgery. Pak Armed Forces Med J 2021; 71(5): 1611-1614. doi: https://doi.org/10.51253/pafmj.v71i5.6856

This is an Open Access article distributed under the terms of the Creative Commons Attribution License (https://creativecommons.org/licenses/by-nc/4.0/), which permits unrestricted use, distribution, and reproduction in any medium, provided the original work is properly cited.

\section{INTRODUCTION}

Visual impairment is a global health burden affecting over two billion people world-wide and is associated with increased morbidity in many countries. Cataract was reported to be the leading cause of blindness in people aged 50 years and older affecting over 15 million (45.5\%) individuals globally. ${ }^{1}$ Cataract is characterized by hardening and clouding of the normally transparent crystalline lens which leads to partial or complete visual loss. It can be classified on the basis of etiology; morphology; age of onset and maturity: immature, mature, intumescent, hyper mature and morgagnian. ${ }^{2,3}$

The corneal endothelial cell count declines with age with a rate of attrition of around $0.4 \%$ every year from the normal cell count of $2000-2500$ cells $/ \mathrm{mm}^{2}$. The long term post-operative transparency of cornea depends on morphological stability and functional integrity

Correspondence: Dr Hussnain Abbas, Classified Eye Specialist, Combined Military Hospital, Multan Pakistan

Received: 08 Jun 2021; revision received: 20 Sep 2021; accepted: 18 Oct 2021 of the corneal endothelium. ${ }^{4}$ Advancements in in cataract surgery technology have reduced the incision size, phacoemulsification energy used and endothelial cell loss thereby improving the phacoemulsification efficiency. ${ }^{5}$ Divide and conquer technique, is the parent nucleo-fracture technique used in phacoemulsification as it provides a safer surgery with less endothelial cell loss. Moreover, it is reliable, simple to learn and serves as a good fall back procedure. ${ }^{6}$ Phacoemulsification procedure can still lead to significant loss of corneal endothelial cell density during surgery leading to prolonged corneal edema. ${ }^{7}$ It is well established through research that some degree of post-operative endothelial cell damage is inevitable. In severe cases, corneal decompensation may occur associated with decreased vision necessitating subsequent corneal transplantation. ${ }^{8}$ However, studies carried out on the effect of phacoemulsification on corneal thickness and endothelial cell loss have shown conflicting figures. Some studies report a risk of severe corneal endothelial cell loss with phacoemulsification in patients with dense cataracts $(52.6 \%)$, whereas others report more favorable figures, estimating rate 
of loss of endothelial cells after the procedure to be between 1.2 and $12 \% .{ }^{9}$ The newer methods of cataract surgery such as femto-laser assisted cataract surgery is considered to be much safer with more consistent refractive outcomes. ${ }^{10}$

Phacoemulsification cataract surgeries have also evolved due to the advancements in Ophthalmic Viscosurgical Devices (OVD) with viscoelastic OVDs introduced mor recently. Nonetheless, divide and conquer method is still a more popular and common technique of phacoemulsification for cataract surgery used in developing countries like Pakistan. Considering the wide prevalence of cataract and its adverse effects in developing countries and a rapidly aging population, this problem is predicted to multiply in future. The aim of the study was to compare the impact of cataract type on variation in the Central Corneal Thickness by divide and conquer technique of phacoemulsification as assessed pre-operatively and at two post-operative timings. METHODOLOGY

This prospective, comparative study was conducted in Combined Military Hospital, Multan from June 2020 to April 2021. Consecutive sampling was used for recruiting patients for this study. All the patients who reported to the Eye Department for a Cataract Surgery were invited to participate in the study. The sample size was calculated using the WHO sample size calculator, using values from the study by Kong-sap. Pre-operative and post-operative central corneal thickness values were used for the sample size calculation. For a 5\% level of significance with $90 \%$ power, with the population mean of $544.2 \pm 45 \mu \mathrm{m}$ and an anticipated mean of $562.5 \pm 42 \mu \mathrm{m}$, a sample size of 64 was calculated. ${ }^{11,12}$ Patients were screened as per a predetermined selection criteria.

Inclusion Criteria: All the patients between the ages of 40 and 70 years with a senile cataract were included.

Exclusion Criteria: Individuals with any corneal degeneration; corneal opacity; a dilated pupil size of $<7 \mathrm{~mm}$; diabetes mellitus; pseudoexfoliation syndrome; traumatic cataract; glaucoma; complicated cataract or having a history of any previous ocular surgeries were excluded from the study.

Those who gave their informed, written consent were included. At the pre-operative assessment appointment, the cataract classification was conducted. The ICD-10 anomaly classification system (ACS) classifies cataract as per different criteria. On the basis of maturity, cataracts are classified as immature, mature, intumescent, hyper mature or morgagnian.,3 For simplifying data analysis, we categorized the cataract status of our patients as immature, mature and hyper mature.

A proforma was used to record a detailed medical history and demographic details of each patients. Phacoemulsification cataract surgery was the intervention employed for all patients. Patients were placed in a supine position. Using a 2.75 keratome ophthalmic knife, traditional phacoemulsification surgery was performed with the 'divide and conquer' technique. A $2 \%$ Methyl Cellulose Dispersive Viscoelastic was used as and when required. Phaco parameters used were: Phaco-I: vacuum $70 \mathrm{mmHg}$, power $60-80 \%$ flow rate $26 \mathrm{ml} / \mathrm{min}$ and Phaco-II: vacuum $350 \mathrm{mmHG}$, power $60-80 \%$ and flow rate $38 \mathrm{ml} / \mathrm{min}$.

For each patient, central corneal thickness was measured at the following appointments: pre-operative; three days post-operative and one-month postoperative. Non contact Tono-pachymeter was used for the measurement of central corneal thickness.

All data was entered and analyzed using SPSS-26. Frequencies and percentages were described for categorical data such as gender and cataract classification. Mean and standard deviation was described for quantitative variables such as age and central corneal thickness. In order to compare the mean central corneal thickness between the different cataract types at the three time intervals (pre-operative; three-day post-operative and one-month post-operative) repeated measures Analysis of Variance (ANOVA) was conducted. The $p$ value of $<0.05$ was considered to be significant.

\section{RESULTS}

A total of 89 patients were included in the study. The mean age of the participants was $62.15 \pm 12.40$ years. There were $49(55.1 \%)$ male and $40(44.9 \%)$ female patients. The gender distribution for each cataract type has been illustrated in the Figure.

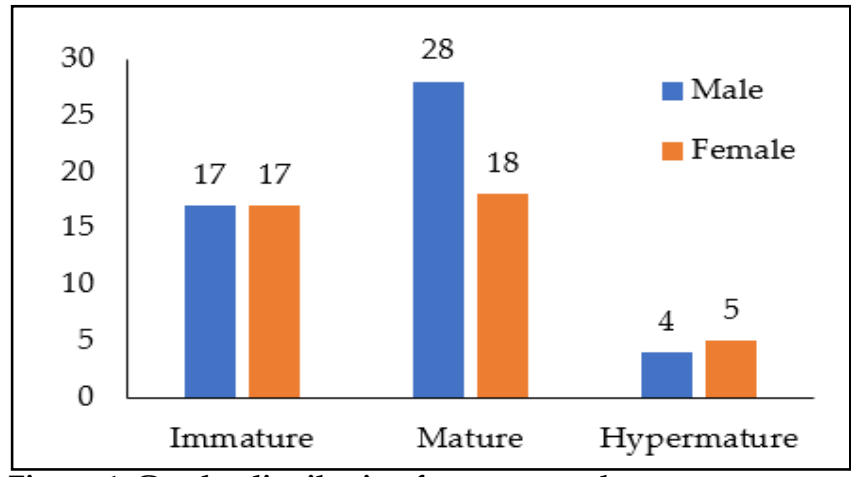

Figure-1: Gender distribution for cataract subtypes. 
The central corneal thickness values for the different types of cataract for the three time intervals have been shown in Table-I. The statistical analysis had a $p$-value $=0.7$, suggesting that the type of cataract was not a significant dependent variable for the variability in central corneal thickness over the three time intervals. In addition, the type of cataract had a partial ETA squared value of 0.027 for the central corneal thickness values. This suggests that the type of cataract only explains $2.7 \%$ of the variability in the central corneal thickness values over the three time intervals. different types of cataracts at the three time inter-vals were not found to be significant ( $p=0.7)$.

Moreover, the results suggested that the central corneal thickness significantly increased from the preoperative stage $(0.48 \pm 0.04 \mathrm{~mm})$ to the three-day postoperative stage $(0.51 \pm 0.04 \mathrm{~mm}, p<0.001)$. Thereafter, the central corneal thickness reported a significant decrease from the third post-operative day till the onemonth post-operative follow up appointment $(0.49 \pm$ $0.04 \mathrm{~mm}, p=0.01)$. However, the difference in central

Table-I: Mean central corneal thickness for different cataract types measured at 3 time intervals.

\begin{tabular}{l|c|c|c|c|c|}
\hline \multirow{2}{*}{ Parameter } & \multirow{2}{*}{ Reference Time } & \multicolumn{3}{|c|}{ Type of Cataract } & \multirow{2}{*}{$\boldsymbol{p}$-value } \\
\cline { 3 - 6 } & & Immature & Mature & Hyper Mature & 0.198 \\
\hline \multirow{2}{*}{$\begin{array}{l}\text { Central Corneal } \\
\text { Thickness (mm) }\end{array}$} & Pre-Operative & $0.49 \pm 0.04$ & $0.48 \pm 0.03$ & $0.47+0.03$ & 0.146 \\
\cline { 2 - 6 } & 3 Days & $0.51 \pm 0.05$ & $0.50 \pm 0.04$ & $0.52+0.03$ & $0.48+0.03$ \\
\cline { 2 - 6 } & 1 Month & $0.49 \pm 0.05$ & $0.49 \pm 0.04$ & 0.551 \\
\hline
\end{tabular}

The analysis was repeated for comparing central corneal thickness at the three time intervals without considering the type of cataract as a dependent variable (Table-I). The mean pre-operative central corneal thickness was $0.49 \pm 0.04 \mathrm{~mm}$. The mean post-operative central corneal thickness values were $0.51 \pm 0.05 \mathrm{~mm}$ at three days and $0.49 \pm 0.05 \mathrm{~mm}$ at one-month. As shown in Table-II, the central corneal thickness values increased significantly by $0.022 \mathrm{~mm}(p<0.00)$ from the preoperative appointment to the third day post-operative. Thereafter, the central corneal thickness significantly decreased from the third day post-operative to onemonth post-operative by $0.018 \mathrm{~mm}(p=0.011)$. However, the difference in central corneal thickness between the pre-operative stage and one-month post-operative $(0.018 \mathrm{~mm})$ was not statistically significant $(p=0.75)$.

Table-II: Comparison of central corneal thickness values at the 3 time intervals (repeated measures ANOVA Test).

\begin{tabular}{l|c|c}
\hline Reference Time & Comparison Time & $\boldsymbol{p}$-value \\
\cline { 2 - 3 } Pre-Operative & 3 Days & 0.001 \\
\cline { 2 - 3 } & 1 Month & 0.759 \\
\hline 3 Days & 1 Month & 0.011 \\
\hline
\end{tabular}

\section{DISCUSSION}

The present study set out to compare the impact of cataract type on variation in the central corneal thickness as assessed pre-operatively and at two postoperative timings.

The results of our study suggest that type of cataract is not a significant predictor of variation in the central corneal thickness. The type of cataract only accounted for $2.7 \%$ of the variability in the central corneal thickness between pre-operative and post-operative stages. The central corneal thickness values between the corneal thickness between the pre-operative appointment and the one-month post-operative appointment was not significant $(0.004 \mathrm{~mm}, p=0.75)$. This suggests that although the central corneal thickness significantly increases right after the surgery, it gradually settles down to pre-operative levels by the one-month postoperative stage. ${ }^{12}$

In a study conducted by Bamdad et al, on changes in corneal thickness and corneal endothelial cell density after phacoemulsification cataract surgery, the mean central corneal thickness was reported to increase by $1.8 \%$ from pre-operative values of $530.47 \pm 2.60 \mathrm{~mm}$ to $540.91 \pm 36.07 \mathrm{~mm}$ post-operatively. The significant increase in postoperative corneal thickness is strongly associated with clinically significant endothelial cell loss. ${ }^{13}$ In a similar study conducted by Perone $e t$ al, the mean central corneal thickness increased by $8.39 \%$ (46.68 \pm $10 \mu \mathrm{m} 2$ hours after surgery and decreased to $1.8 \% 10 \pm$ $18 \mu \mathrm{m} 4$ days after surgery and only left an increase of $0.1 \% 0.76 \pm 11.4 \mu \mathrm{m} 15$ days after surgery. ${ }^{4}$ The results of both these studies are in agreement with our study findings.

Louis et al compared effect of central corneal thickness in phacoemulsification surgeries performed by experienced and inexperienced surgeons. The twohour post-operative change in central corneal thickness performed by inexperienced surgeons (mean increase in central corneal thickness $=105.8 \pm 81.4 \mu \mathrm{m}$ ) was found to be significantly greater than in surgeries done by experienced surgeons (mean increase in central corneal thickness $=66.4 \pm 3.7 \mu \mathrm{m}, p=0.001$ ). Although, the experience of the surgeon was reported to be a significant factor in causing increase in central corneal thickness 


\section{Cataract Type on Central Corneal Thickness}

and edema in this study, long term follow-up results were not reported. It would have been interesting to compare the results of both groups after one week and one month of the surgery. ${ }^{14}$

Wali et al, compared the central corneal thickness levels of 93 patients at four time intervals: pre-operative; post-operative first day, one week and one month. Similar to our study, the central corneal thickness increased right after the surgery. However, the central corneal thickness levels subsided back to pre-operative levels $(497.65 \mu \mathrm{m})$ after one month post-operatively (502 $\mu \mathrm{m} ; p<0.05) .{ }^{15}$ Similarly, Simova et al also compared the central corneal thickness levels of 60 patients at four time intervals: pre-operative, post-operative day 1, day 7 and day 30. Although the central corneal thickness levels increased after the surgery, they subsided back to pre-operative levels $(552 \mu \mathrm{m})$ after 30 days post-operatively $(566 \mu \mathrm{m}) .^{16}$

In accordance with our finding, a study conduc-ted by Ventura et al, on corneal thicknes s and endo-thelial density before and after cataract surgery repor-ted that central corneal thickness raised from pre-op (0.52-0.56 $\mathrm{mm}$ as measure by optical low coherence ref-lectometry (OLCR)) to post-op; one day after surgery $(0.52-0.72 \mathrm{~mm}$ as measured by ultrasonic pachymetry) but it returned to the pre-op values $(0.5-0.56 \mathrm{~mm}$ measured by OLCR) 3 months after surgery. ${ }^{17} \mathrm{In}$ fact, the corneal thickness is reported to return to the pre-operative values within 4 weeks of cataract surgery. ${ }^{12,15,16,18,19}$

Modern cataract surgery is associated with minimal complications owing to the use of OVDs. OVDs protect the corneal epithelium, in addition to releasing oxygen free radicals thereby facilitating the surgical procedure and reducing damage to intraocular structures. $^{20}$

\section{CONCLUSION}

Our study did not find any association between the type of cataract and the central corneal thickness. However, central corneal thickness was found to significantly increase right after the surgery. The central corneal thickness levels, however, do subside back to pre-operative level in about one month's time. Therefore, it is recommended that post-operative refraction should be finalized after one month of the surgery. In addition, the post-operative treatment should be continued for at least month after the surgery.

\section{Conflict of Interest: None.}

\section{Authors' Contribution}

HA: Data collection, data analysis, AR: Interpretation of data, SP: Conception of data, proof reading, SHM: Data analysis, IH: Data analysis, KN: Intellectual contribution.

\section{REFERENCES}

1. Steinmetz JD, Bourne RR, Briant PS, Flaxman SR, Taylor HR, Jonas JB, et al. Causes of blindness and vision impairment in 2020 and trends over 30 years, and prevalence of avoidable blindness in relation to VISION 2020: the Right to Sight: an analysis for the Global Burden of Disease Study. Lancet Glob Health 2021; 9(2): e144-e160.

2. Mandelblum J, Fischer N, Achiron A, Goldberg M, Tuuminen R, Zunz E, et al. A Simple pre-operative nuclear classification Score (SPONCS) for grading cataract hardness in clinical studies. J Clin Med 2020; 9(11): 3390 .

3. Thylefors B, Chylack LT, Konyama K, Sasaki K, Sperduto R, Taylor $\mathrm{HR}$, et al. A simplified cataract grading system. Ophthalmic Epidemiol 2002; 9(2): 83-95.

4. Perone JM, Boiche M, Lhuillier L, Ameloot F, Premy S, Jeancolas AL, et al. Correlation between postoperative central corneal thick-ness and endothelial damage after cataract surgery by phacoemul-sification. Cornea 2018; 37(5): 587-590.

5. Jeancolas AL, Lhuillier L, Renaudin L, Boiche M, Ghetemme C, Goetz $\mathrm{C}$, et al. Central corneal thickness assessment after phacoemulsification: Subluxation versus Divide-and-Conquer. J Fr Ophtalmol 2017; 40(9): 744-750.

6. Ali MA, Darasheni S, Rub FU, Ahmed I, Zafar MU. Comparison between different techniques of phaco emulsification divide and conquer and horizontal chop. Pak J Surg 2020; 36(2): 190-194.

7. de Silva SR, Riaz Y, Evans JR. Phacoemulsification with posterior chamber intraocular lens versus extracapsular cataract extraction (ECCE) with posterior chamber intraocular lens for age-related cataract. Cochrane Database Syst Rev 2014; 1(1): CD008812.

8. Gogate P. Comparison of various techniques for cataract surgery, their efficacy, safety, and cost. Oman J Ophthalmol 2010; 3(3): 105-112.

9. Rosado-Adames N, Afshari NA. The changing fate of the corneal endothelium in cataract surgery. Curr Opin Ophthalmol 2012; 23(1): 3-6.

10. Charles Crozafon P, Bouchet C, Zignani M, Griner R, Foster SD, Zou $\mathrm{M}$, et al. Comparison of real-world treatment outcomes of femtosecond laser-assisted cataract surgery and phacoemulsifi-cation cataract surgery: A retrospective, observational study from an outpatient clinic in France. Eur J Ophthalmol 2020; 1(1): 1120672120925766.

11. Arshinoff SA, Jafari M. New classification of ophthalmic visco-surgical devices-2005 J Cataract Refract Surg 2005; 31(11): 2167-2171.

12. Kongsap P. Central corneal thickness changes following manual small incision cataract surgery versus phacoemulsification for white cataract. Rom J Ophthalmol 2019; 63(1): 61-66.

13. Bamdad S, Bolkheir A, Sedaghat MR, Motamed M. Changes in corneal thickness and corneal endothelial cell density after pha-coemulsification cataract surgery: a double-blind randomized trial. Electron Phys 2018; 10(4): 6616-6622.

14. Lhuillier L, Jeancolas AL, Renaudin L, Goetz C, Ameloot F, Premy S, et al. Impact of ophthalmic surgeon experience on early post-operative central corneal thickness after cataract surgery. Cornea 2017; 36(5): 541-545.

15. Wali FS, AliSurhio S, Talpur R, Jawed M, Shujaat S. Change in Central corneal thickness after Phacoemulsification. Pak J Ophthalmol 2020; 36(1): 67-71.

16. Simova J, Radeva M, Grupchev D, Mihova T. Central corneal thickness and morphological changes in the cornea after uneventful phacoemulsification. Bulg Rev Ophthalmol 2018; 62(4): 10-17.

17. Ventura AS, Wälti R. Corneal thickness and endo-thelial density before and after cataract surgery. Br J Ophthalmol 2001; 85(1): 18-20.

18. Cheng H, Bates AK, Wood L, McPherson K. Positive correlation of corneal thickness and endothelial cell loss: serial measurem-ents after cataract surgery. Arch Ophthalmol 1988; 106(7): 920-922.

19. Kohlhaas M, Stahlhut O, Tholuck J, Richard G. Changes in cor-neal thickness and endothelial cell density after cataract extrac-tion using phacoemulsification. Ophthalmol 1997; 94(7): 515-518.

20. Malvankar-Mehta MS, Fu A, Subramanian Y, Hutnik C. Impact of ophthalmic viscosurgical devices in cataract surgery. J Ophthalmol 2020; 1(1): 1-17. 